\title{
Characterization of the JWST Pathfinder mirror dynamics using the center of curvature optical assembly (CoCOA)
}

\author{
Conrad Wells ${ }^{(* a)}$, James B. Hadaway ${ }^{\text {, }}$ Gene Olczak ${ }^{\mathrm{a}}$, Joseph Cosentino ${ }^{\mathrm{a}}$, John D Johnstone, Tony Whitman ${ }^{\mathrm{a}}$, Mark \\ Connollya , David Chaneyc, J. Scott Knightc \& Randal Telfer ${ }^{\mathrm{d}}$ \\ ${ }^{a}$ Harris Corporation, 800 Lee Rd., Rochester, NY 14606 \\ ${ }^{\mathrm{b}}$ University of Alabama in Huntsville, 301 Sparkman Dr., Huntsville, AL 35899 \\ 'Ball Aerospace \& Technologies Corp., 1600 Commerce St., Boulder, CO 80301 \\ dSpace Telescope Science Institute, 3700 San Martin Dr., Baltimore, MD 21218 \\ eNASA, Goddard Space Flight Center, Greenbelt Road, Greenbelt, MD 20771
}

\begin{abstract}
The JWST Optical Telescope Element (OTE) consists of a $6.6 \mathrm{~m}$ clear aperture, 18 segment primary mirror, all-reflective, three-mirror anastigmat operating at cryogenic temperatures. To verify performance of the primary mirror, a full aperture center of curvature optical null test is performed under cryogenic conditions in Chamber A at NASA Johnson Space Center using an instantaneous phase measuring interferometer ${ }^{123}$. After phasing the mirrors during the JWST Pathfinder testing, the interferometer is utilized to characterize the mirror relative piston and tilt dynamics under different facility configurations. The correlation between the motions seen on detectors at the focal plane and the interferometer validates the use of the interferometer for dynamic investigations. The success of planned test hardware improvements will be characterized by the multi-wavelength interferometer (MWIF) at the Center of Curvature Optical Assembly (CoCOA).
\end{abstract}

Keywords: James Webb Space Telescope, OTIS, optical alignment, photogrammetry, interferometer, multi-wavelength

\section{INTRODUCTION}

After integration of the Optical Telescope Element (OTE) to the Integrated Science Instrument Module (ISIM) to become the OTIS, the James Webb Space Telescope OTIS is tested at NASA's Johnson Space Center (JSC) in the cryogenic vacuum Chamber A for alignment and optical performance. Figure 1 illustrates the test configuration and the optical paths involved in the testing. Hardware unique to this test configuration include the 4D Technologies instantaneous phase shifting multi-wavelength interferometer (MWIF) ${ }^{4}$ and Harris-patented all reflective null optics ${ }^{56}$.

The alignment of the mirrors comprises a sequence of steps as follows ${ }^{2,7}$ : The mirrors are coarsely aligned using photogrammetry cameras with reflective targets attached to the sides of the mirrors. Then a multi-wavelength interferometer is aligned to the 18-segment primary mirror using coarse and fine alignment cameras at the center of curvature $^{8}$. Once the interferometer is aligned, the 18 primary mirror segments are then adjusted to optimize wavefront error of the aggregate mirror. This process aligns the mirrors in 6 degrees of freedom plus radius of curvature.

Vibration contributes uncertainty in the primary mirror figure measurement with movement between mirror segments and variation in the alignment between the mirror and the interferometer. Temporal variations in the relative positions of the mirror segments are manifested as primary mirror figure error. The variation in the alignment of the mirror relative to the interferometer adds fringe density contributing to measurement noise and, potentially, aliasing. The multiple-wavelength interferometer can align and phase the mirror segments with step heights between segments of more than one millimeter by combining phase maps of differing wavelengths to remove phase ambiguity. Movement of the mirrors between phase maps also adds uncertainty in the phasing of the segments. To limit the impacts of vibration on the primary mirror wavefront measurement, the interferometer takes 100 or more instantaneous frames and averages the results. 

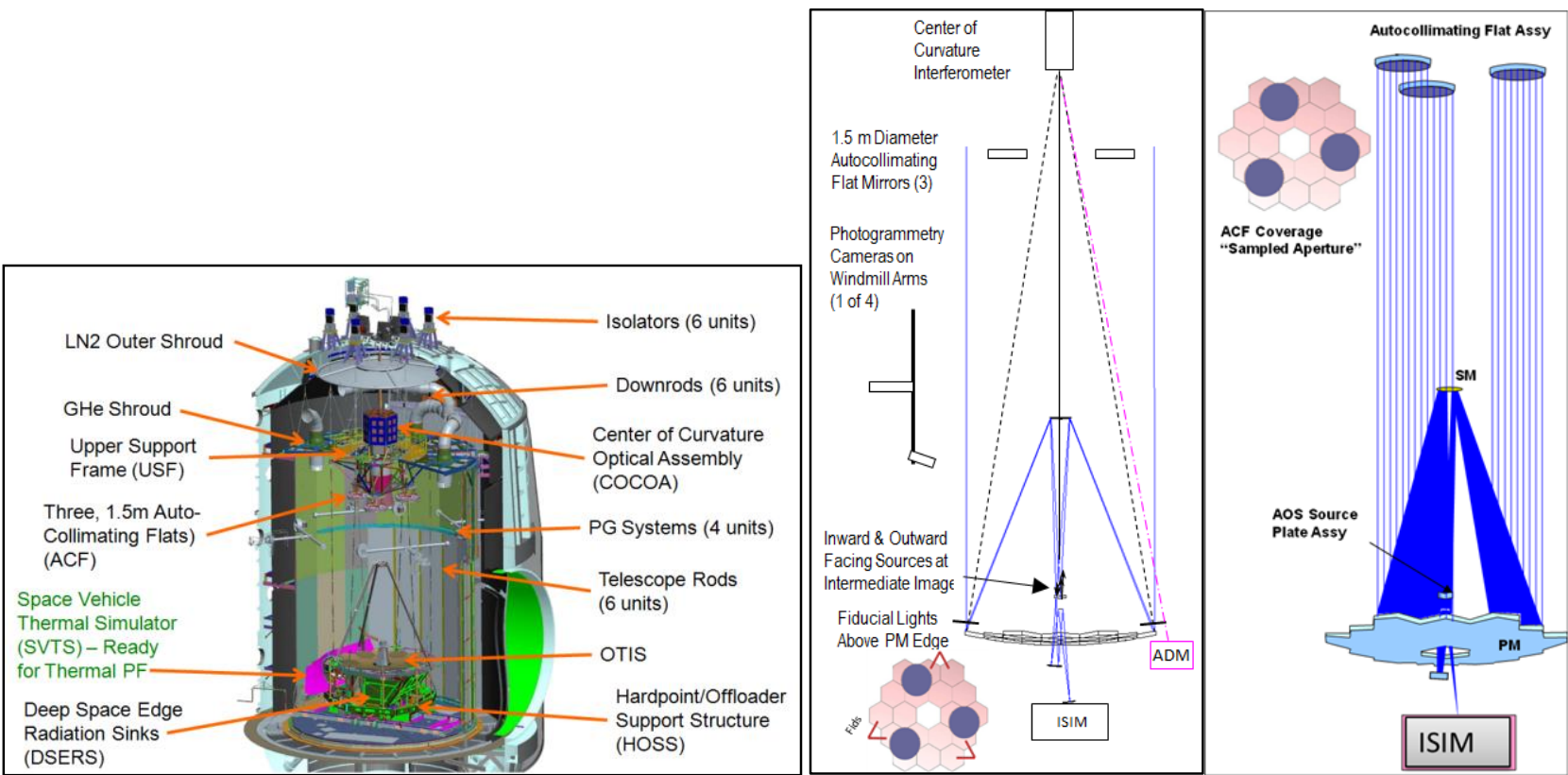

Figure 1 Illustrations of the mechanical and optical configurations of the JWST test configuration. The entire configuration is supported from the top of the chamber by a vibration isolation system. The center of curvature interferometer, Cassegrain sources on the AOS (aft optics subsystem) Source Plate Assembly and autocollimating flats are shown along with the optical paths.

To test the observatory as a whole, an optical fiber placed at the Cassegrain focus of the telescope emits light towards the secondary mirror to create a collimated beam emitting from the primary mirror. Portions of the collimated beam are retroreflected from flat mirrors at the top of the chamber to pass through the telescope to the SI detector. These optical fiber sources are used to produce simulated star point spread functions (PSF) at the detector once imaged through the OTE during PAAH testing. The imaging team processes these images utilizing wavefront sensing techniques ${ }^{11}$. The image on the detector is used for fine alignment of the secondary mirror and a check of the primary mirror alignment using many of the same analysis techniques used in the on-orbit alignment.

The wave front sensing techniques require a stable PSF because mirror to mirror vibrations can cause phase ambiguities in the wave front reconstruction. The vibration in the testing chamber comes from the ground, the surrounding facility infrastructure to support the cryogenic test, and a clean room directly adjacent to the chamber. Chamber A at the Johnson Space Center is one of the few cryogenic vacuum chambers large enough for the JWST optical test. The chamber was designed and built for testing of the hardware and personnel of the Apollo mission in the 1960's rather than optimized for a 21st century interferometer test - complete with operating vacuum pumps and gaseous helium cryogenics. The ground vibrations are minimized with modifications to the test facility, and by the architecture of the equipment supporting the load $^{9}$. Additional special test equipment (including strategically placed isolators, tunable mass dampers, and cryogenic magnetic dampers) mitigates the vibration and the response sensitivity before reaching the telescope. Some shorts to ground are present such as cryogenic cooling and electrical lines.

Nonetheless, measurable vibration still impacts the optical testing. To characterize the dynamic environment, focal plane images as well as interferometer and accelerometer data is acquired when air handlers, cryogenic systems, or vacuum systems are powered down for brief periods during test.

\section{MIRROR PHASING WITH THE MULTI-WAVELENGTH INTERFEROMETER}

The multi-wavelength interferometer is utilized to negate the effects of vibration during primary mirror alignment and surface figure characterization. These measurements consist of 100 or more frames (each "frame" consisting of two fringe 
images, one at each wavelength) of data which are averaged to create a wave front map of the mirror segments. The Segmented Aperture Software Suite (SASS) ${ }^{10}$ software both processes interferometric data at a single wavelength, and mathematically combines the wavefronts from the two wavelength interferograms into a synthetic wavefront (designed to make the data appear to have been taken at a much larger wavelength, ranging from $16.8 \mu \mathrm{m}$ to $15 \mathrm{~mm}$ ) in order to be able to measure mm-class step heights between. Repeatable measurements are achieved by averaging out the impacts of vibration. The synthetic wavelength utilized during fine phasing and dynamics characterization is $16.8 \mu \mathrm{m}$, enabling interferometric accuracy on step heights exceeding $1 \mu \mathrm{m}$ in height. This synthetic wavelength consists of two fringe images at $687 \mathrm{~nm}$ and $660 \mathrm{~nm}$ wavelength.

The relative alignment between the two Pathfinder segments at cryogenic temperatures achieved a vertical spacing position difference of $32 \mathrm{~nm}$ (Figure 2). The demonstrated WFE measurement repeatability is $11 \mathrm{~nm}$ RMS with a segment to segment piston repeatability of $7 \mathrm{~nm}$ and a tilt repeatability of $6.5 \mathrm{nrad}$ per axis.

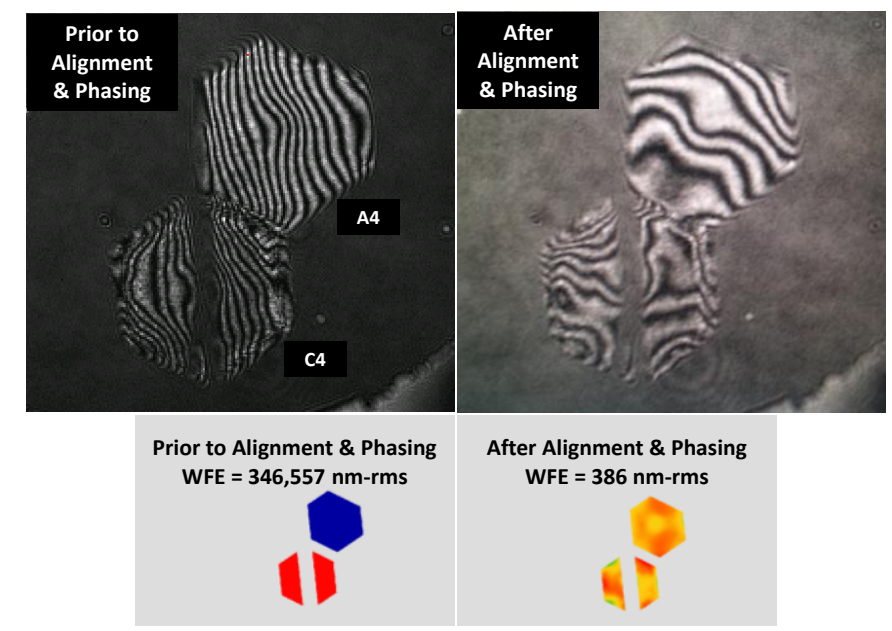

Figure 2 PMSAs aligned to a vertical (along perpendicular to optical surface) spacing of $32 \mathrm{~nm}$.

\section{PATHFINDER TESTING}

The primary mirror and PAAH pathfinder tests in 2015 were the first time the OTE design had been tested as an optical system along with the ground support equipment (GSE). The pathfinder is similar but not identical to the flight system. The key differences are that the primary has only two primary mirror segments in the A4 and $\mathrm{C} 4$ positions, the pathfinder backplane has just a portion of the flight backplane, and the science camera is replaced by a camera on a 3-axis stage to measure images at the telescope focal surface. Figure 3 shows a cartoon of the two segments in yellow as compared to full 18-segment primary mirror and sampled by a single subaperture auto-collimating flat (ACF). Figure 3 also shows a photograph of the pathfinder system in the vacuum chamber where the two segments can be seen. Only one segment is coated in gold and the other segment is uncoated Beryllium. Phasing of the two segments as well as multi-field wave front sensing is performed to test the optical ground support equipment, reduce risk, train staff, and to validate the facility performance. 


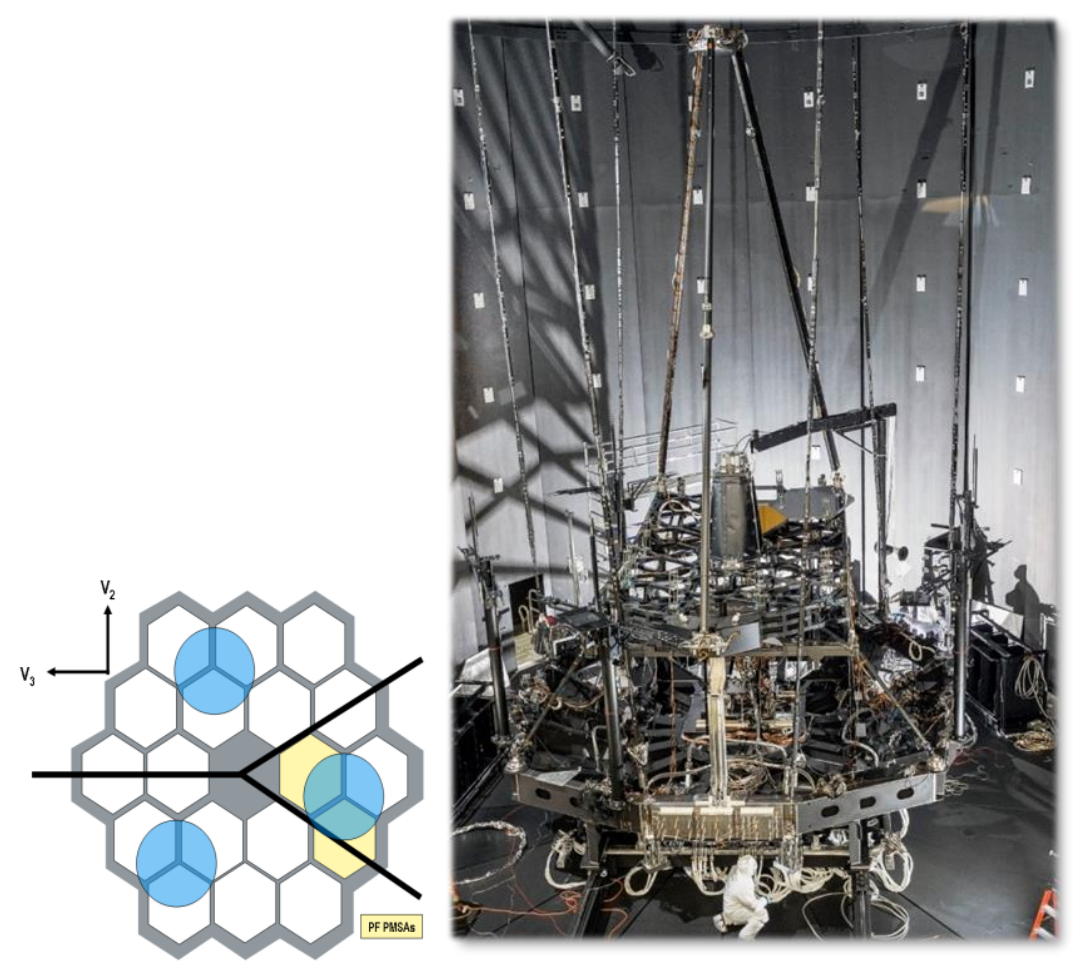

Figure 3 The layout of the ACF subaperture sampling of the pupil relative to the primary mirror. The pathfinder test had only two of the 18 segments installed as shown in yellow. To the right is a picture of the pathfinder test article inside the Johnsons Space Flight Center Chamber A.

\section{VIBRATION CHARACTERIZATION FOR INTERFEROMETRY}

Much of what makes the MWIF unique is the dual wavelength measurement capability. The MWIF has three sources in all, two fixed-wavelength lasers and one tunable wavelength laser, of which two are used at a time for a single syntheticwavelength measurement at 2.2 frames per second. The MWIF uses a clever timing scheme so that two measurements, at different wavelengths, occur in adjacent sequential image frames so that non-common errors due to vibration are minimized. The data from these two measurements in combination is treated as a synthetic wavelength measurement. For a pair of fundamental wavelengths $\lambda_{1}$ and $\lambda_{2}$ the synthetic wavelength $\lambda_{s}$ is given by

$$
\lambda_{s}=\frac{\lambda_{1} \cdot \lambda_{2}}{\left|\lambda_{2}-\lambda_{1}\right|}
$$

and the phase map of $\lambda_{s}$ is determined from the difference of the phase maps generated at $\lambda_{1}$ and $\lambda_{2}$.

To generate the multi-frame (typically 100) average ${ }^{10}$, each fringe image is masked and the raw phase is calculated for each wavelength. The raw phase between the two wavelengths is subtracted to calculate the phase at the synthetic wavelength. After unwrapping the synthetic wavelength for a given pair of fringe images (that have the dynamic range required), the fundamental wavelength $(687 \mathrm{~nm}$ ) phase image is unwrapped and the different island heights (segments or pieces of segments separated by a strut, Figure 2) are seeded with values from the synthetic wavelength data. This process ensures an unambiguous $687 \mathrm{~nm}$ wavefront map for each of the 100 frames. Each phase map is processed by a pose estimator ${ }^{10}$ to calculate the rigid body motion for each mirror segment with the 7 degrees of freedom to minimize RMS WFE. The statistics of the 100 frames provide a measure of the dynamics during a measurement.

Figure 4 illustrates histograms of the wavefront piston values (in units of waves) for a measurement under ambient vacuum conditions. At ambient, the chamber vibrations are reduced and the transmission of vibration to the Pathfinder is greatly dampened. 
For the segment heights to be unambiguously measured at the synthetic wavelength, the total dynamic range of the wave front piston variation from measurement to measurement must be less than $+/-0.25 \lambda(+/-4.2 \mu \mathrm{m}$ in all sources of variance and bias in the data.) This is a function of both the step height and the dynamics. The synthetic wavelength $(16.8 \mu \mathrm{m})$ plot (top histogram in Figure 4) has an $\mathrm{x}$ axis range of $+/-0.015 \lambda$ and represents the measured step values. The histograms are used to quantify whether the measurement has the dynamic range required to measure the steps as well as to quantify the variation in the measured piston values. The variation is Gaussian in nature $(1 \sigma=36 \mathrm{~nm})$ with a peak to valley range in wavefront piston values of $195 \mathrm{~nm}$. The measured wave front step height is $103 \mathrm{~nm}$.

Once seeded by the synthetic wavelength the variation of the measured step height (from the average value) at the fundamental wavelength $(687 \mathrm{~nm})$ must also be less than $+/-0.25 \lambda$. The $687 \mathrm{~nm}$ histogram represents the delta WFE piston between each frame and the 100 frame average (Note this is a slightly different metric than the 16.8 um histogram). The peak to valley variation in wavefront piston values is $195 \mathrm{~nm}$ and the measured wave front step height is $116 \mathrm{~nm}$. The variation Gaussian in nature $(1 \sigma=28 \mathrm{~nm})$ and the step is properly resolved without ambiguity. The agreement between the $687 \mathrm{~nm}$ and the $16 \mathrm{um}$ wavelength is $13 \mathrm{~nm}$ !

The measurements at ambient vacuum demonstrate the absolute agreement between the synthetic and fundamental wavelengths and the mirrors were successfully phased using both the synthetic wavelength and the seeded fundamental wavelength.
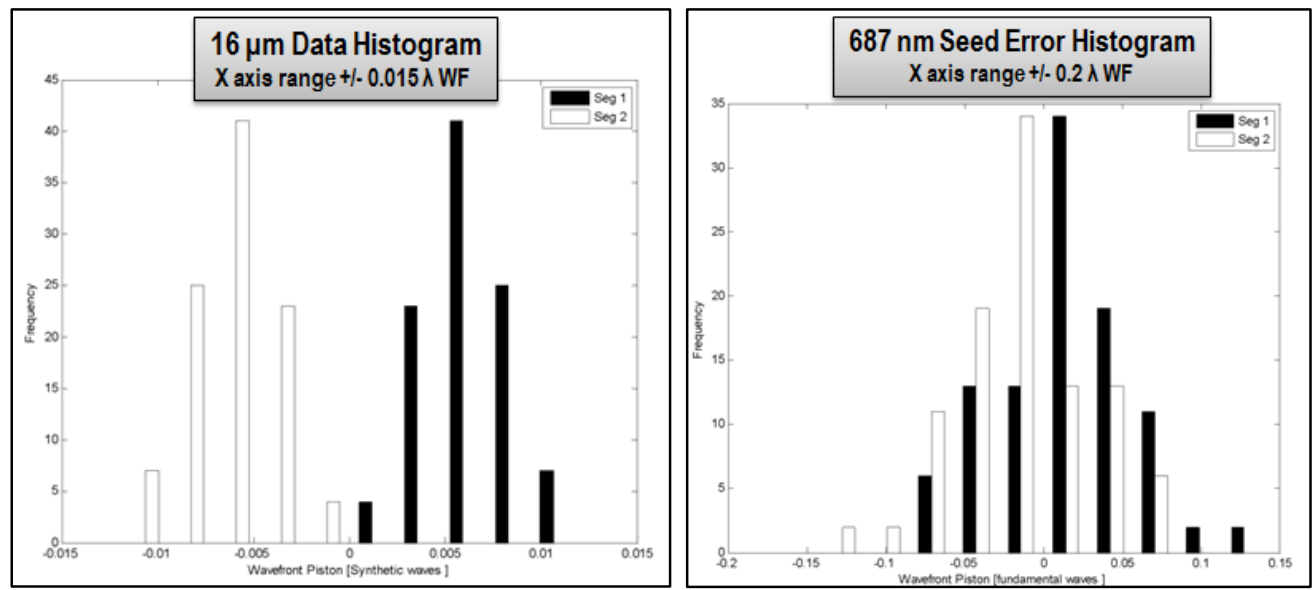

Figure 4 Histogram of segment level piston values for the synthetic and fundamental wavelengths under vacuum conditions

Figure 5 illustrates the results for testing under stable cryogenic vacuum conditions. Under these conditions, the chamber vibration increases and the damping of the vibration transmitted to the telescope is greatly reduced. Phasing is successfully demonstrated using the 16.8 um synthetic wavelength, but the dynamic environment causes the fundamental wavelength to wrap because the amount of vibration exceeded $+/-1 / 4 \lambda$. The peak to valley vibration at the synthetic wavelength is $3.3 \mathrm{x}$ the value under ambient conditions, but the range is only $+/-0.015 \lambda$ so the measurements are resolved. In this case the synthetic wavelength is utilized to measure the step height, the accuracy of which is previously proven. Repeated back to back measurements at cryo demonstrated 100 frame average segment to segment wave front piston repeatability of 14 nm. 

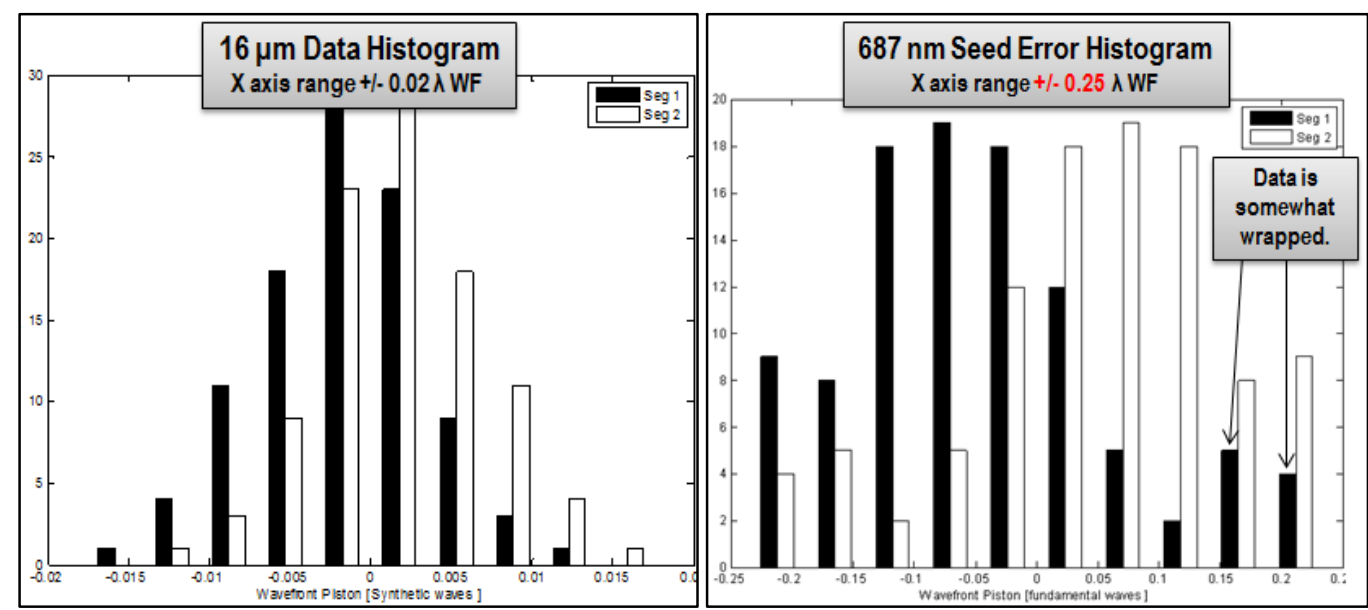

Figure 5 Histogram of segment level piston values for the synthetic and fundamental wavelengths under cryogenic vacuum conditions

Table 1 summarizes the dynamics of the primary mirrors as measured by the interferometer during two testing cycles, OGSE 1 and OGSE 2. Isolation system improvements made between the tests reduces the piston vibration between the segments, along with a reduction in the peak to valley tilt variation.

\begin{tabular}{|c|c|c|c|c|c|}
\hline & \multicolumn{5}{|c|}{ Observed Vibration During OGSE Testing } \\
\hline & OGSE 1 & OGSE 2 & \multirow{2}{*}{$\begin{array}{c}1 \text { to } 2 \text { Vibe } \\
\text { Improvement }\end{array}$} & \multirow{2}{*}{$\begin{array}{c}\text { OGSE } 2 \\
\text { Ambient } \\
\end{array}$} & \multirow{2}{*}{$\begin{array}{l}\text { Cryo to Ambient } \\
\text { Improvement }\end{array}$} \\
\hline & Cryo & Cryo-157 & & & \\
\hline Stdev Surface Piston & $55 \mathrm{~nm}$ & $44 \mathrm{~nm}$ & $-20 \%$ & $18 \mathrm{~nm}$ & $-59 \%$ \\
\hline P/ Surface Piston* & $561 \mathrm{~nm}$ & $251 \mathrm{~nm}$ & $-55 \%$ & $97 \mathrm{~nm}$ & $-61 \%$ \\
\hline PN Surface Waves Syn* & $0.003 w v$ & $0.003 w v$ & & $0.001 \mathrm{wv}$ & \\
\hline PN Surfade Waves Fund* & $0.82 \mathrm{wv}$ & $0.37 \mathrm{wv}$ & & $0.14 w v$ & \\
\hline Stdev Surface Tilt & $261 \mathrm{nrad}$ & $305 \mathrm{nrad}$ & $17 \%$ & $51 \mathrm{nrad}$ & $-83 \%$ \\
\hline \multirow[t]{2}{*}{ P/ Surface Tilt } & $1377 \mathrm{nrad}$ & $1259 \mathrm{nrad}$ & $-9 \%$ & $214 \mathrm{nrad}$ & $-83 \%$ \\
\hline & \multicolumn{5}{|c|}{${ }^{*} \mathrm{P} / \mathrm{N}$ vibration must be $<0.25$ surface, $<0.5$ waves $\mathrm{WF}$} \\
\hline
\end{tabular}

Table 1 Interferometer dynamics summary from the two Pathfinder tests (OGSE 1 and OGSE 2.)

The interferometer demonstrated the ability to average out the vibration in all cases and successfully phased the primary mirror in the presence of vibration that exceeded the pretest predictions. Its ability to measure the mirror to mirror piston and tilt allowed the dynamics team to characterize the actual mirror dynamics under atmospheric, vacuum and cryogenic vacuum conditions.

\section{IMPACTS OF MIRROR VIBRATION ON PAAH WAVE FRONT SENSING ${ }^{11}$.}

The pathfinder test was used to verify the test methodology and GSE of both the center of curvature based mirror phasing as well as the PAAH imaging test. The basic test approach was performed and found to work as expected with one exception. The amount of vibration and resulting image and wavefront jitter in response to the chamber excitation was significantly higher than expected. In order to perform phase retrieval over stacked segment images, the wavefront jitter was expected to be less than $1 / 2$ wave peak-to-valley maximum (excluding global piston and tilt) within an ACF subaperture. The combined line-of-sight jitter (correlated tilt over the full ACF subaperture) met expectations. However, higher than expected wavefront jitter caused by the relative piston and tilt between segments totaled about 5 waves peakto-valley. 

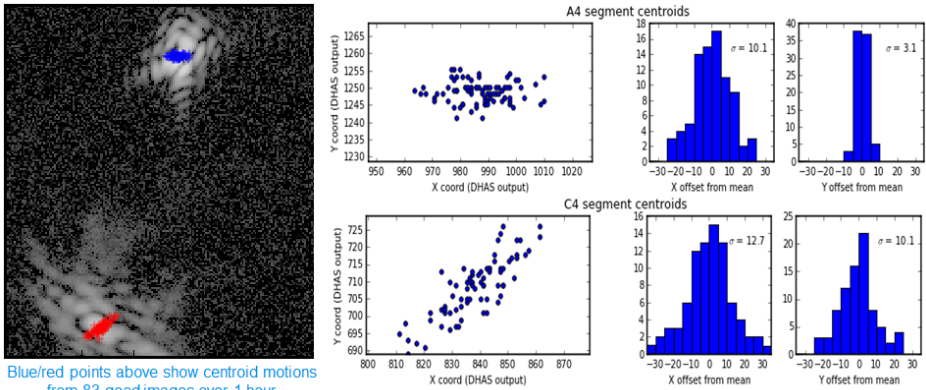

Figure 6 Jitter data observed during the pathfinder test ${ }^{11}$. The top left panel shows an example image of the two segment subimages. The red and blue points show the measured centroid displacement over the data collection. The remaining top panels show the detailed analysis of the centroid data.

\section{CORRELATION BETWEEN INTERFEROMETER AND IMAGE PLANE DYNAMICS}

A comparison of the observed PAAH image motion and motion calculated from the interferometer data (Figure 7) agreed to $\sim 1$ pixel in most cases. The correlation demonstrated that the interferometer can be used to accurately predict the image based jitter. It also confirmed the image motion jitter is dominated by PMSA to PMSA tilt contributions. Further interferometric testing performed by the dynamics team under cryogenic, vacuum and atmospheric conditions allowed the team to quickly assess the impacts of different mechanical features of the test configuration. Dynamics and interferometric data collected as a diagnostic indicates that the vibration is due to an unexpected amount of vibration transmitted from the floor of the chamber through the test ground support equipment configuration, coolant lines and electrical cables.

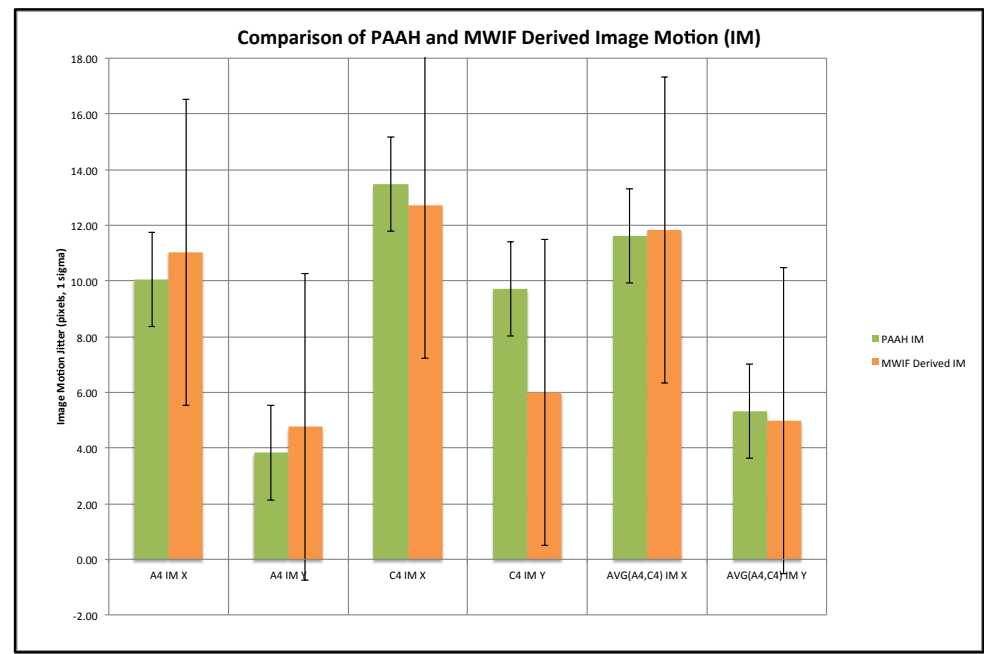

Figure 7 Comparison of the observed image motion and calculated motion from interferometer data.

\section{FUTURE PATHFINDER TESTING}

An additional Pathfinder test is planned in 2016. Isolation system, electrical and cooling line design improvements are made using the critical lessons learned from the previous tests. Because the test does not include the PAAH image based testing, the interferometry data will be used to assess the success of the design upgrades. Once again, testing will be performed under a variety of facility and thermal conditions. 


\section{SUMMARY}

Successful primary mirror phasing is demonstrated during pathfinder testing in the presence of larger than expected dynamic disturbances. The interferometer proved to be a valuable tool to characterize the dynamics environment with a high degree of correlation to image based motion. The quality of the design improvements is tested with the interferometer in the upcoming pathfinder testing. This allows the mechanical and optical teams to predict the performance expected in the OTIS test in 2017.

\section{ACKNOWLEDGEMENTS}

The optical test design, preparation, and execution were supported by the JWST contract NNG11FD64C with NASA GSFC. The JWST system is a collaborative effort involving NASA, ESA, CSA, the Astronomy community and numerous principal investigators. The Pathfinder test and analysis were accomplished through the efforts of NASA Goddard Space Flight Center, NASA Johnson Space Center, Harris Space and Intelligence Systems, Ball Aerospace and Technologies, Genesis Engineering Solutions, the Space Telescope Science Institute and Northrop Grumman.

\section{REFERENCES}

${ }^{1}$ Conrad Wells, et al, The Center of Curvature Optical Assembly for the JWST Primary Mirror Cryogenic Optical Test, Proc. SPIE 7739, 2010

${ }^{2}$ Tony L. Whitman et al. Alignment Test Results of the JWST Pathfinder Telescope Mirrors in the Cryogenic Environment, Proc. SPIE 9904 (2016)

${ }^{3}$ James B Hadaway et al, Performance of the primary mirror center-of-curvature optical metrology system during cryogenic testing of the JWST Pathfinder Telescope Proc. SPIE 9904 (2016)

${ }^{4}$ Michael North-Morris, James E. Millerd, Neal J. Brock, John B. Hayes, Phase-shifting multiwavelength dynamic interferometer, Proc SPIE Vol. 5531, (2004)

${ }^{5}$ Gene Olczak, Conrad Wells, David J. Fischer, Mark T. Connolly, Wavefront calibration testing of the James Webb Space Telescope primary mirror center of curvature optical assembly, Proc. SPIE Vol 8450 (2012)

${ }^{6}$ Olczak, Eugene G. , Hannon, John J., Dey, Thomas W., Jensen, Arthur E., "Optical nulling apparatus and method for testing an optical surface", US Patent 7336370 (2008).

${ }^{7}$ Conrad Wells et al, The center of curvature optical assembly for the JWST primary mirror cryogenic optical test: optical verification Proc SPIE Vol. 7790, (2010)

${ }^{8}$ Sharon Lunt et al, Model predictions and observed performance of JWST cryogenic position

metrology system, Proc. SPIE 9904, (2016)

${ }^{9}$ Tony L. Whitman et al. Measuring segmented primary mirror WFE in the presence of vibration and thermal drift on the light-weighted JWST, Proc. SPIE Vol 8442 (2012)

${ }^{10}$ Gene Olczak et al, James Webb Space Telescope primary mirror integration: testing the multiwavelength interferometer on the test bed telescope, Proc SPIE Vol. 8146, (2011)

${ }^{11}$ Knight, J. S. et al. "Hartmann Test for the James Webb Space Telescope”, Proc. SPIE 9904, (2016) 